\title{
Numerical Simulation of the Jovian Wind Band as a Convective Phenomenon
}

\author{
Kwing L. Chan \\ The Hong Kong University of Science and Technology, Hong Kong, \\ China
}

\author{
Hans G. Mayr \\ Goddard Space Flight Center/NASA, Greenbelt, MD, USA
}

Using a three-dimensional numerical spectral model, we simulate the outermost layer of Jupiter's convective envelope (two depth cases: 1-23 bars, 1-115 bars). The physical parameters (e.g. internal energy flux, rotation rate) are chosen to be close to those expected, but solar heating as well as dynamical influences from deeper layers are ignored. The model generates a wind field pattern remarkably similar to that observed. There is a narrow, super-rotating jet at the equator, and two prominent humps in temperature also develop in the subtropics. The strength of the jet streams does not change much over depth. The maximum wind speed occasionally reach $100 \mathrm{~m} / \mathrm{s}$, but the mean amplitude of the equatorial jet is about a factor of $2-3$ lower than the nominal value. The latitudes of the secondary pro-grade jets are higher than those observed, but they are dependent on the depth of the model. Though the quantitative agreement is not quite satisfactory (as might have been caused by neglected physical effects like solar radiation), this model demonstrates, in principle, the feasibility of generating a Jovian type wind pattern through the interaction of fast rotation and convection in a thin shell. 